\title{
Palladium(II)-Catalyzed Intramolecular Diamination of Unfunctionalized Alkenes
}

Jan Streuff, Claas H. Hövelmann, Martin Nieger and Kilian Muñiz

Kekulé-Institut für Organische Chemie und Biochemie,

Universität Bonn

Gerhard-Domagk-Str. 1,

D-53121 Bonn,

Germany

kilian.muniz@uni-bonn.de

SUPPORTING INFORMATION 


\section{Experimental Section}

\section{General.}

All organic reagents were purchased from Acros. Palladium acetate and i...odosobenzene diacetate were purchased from Aldrich. Dichloromethane, ethyl acetate and hexanes were dried over calcium chloride, distilled and used without further manipulation. Column chromatography was performed with silica gel (Merck, type 60, 0.063-0.2 mm and Machery Nagel, type 60, 0.015-0.025 mm). Nmr spectra were recorded on a Bruker DPX $300 \mathrm{MHz}$ and Bruker DRX $500 \mathrm{MHz}$ spectrometer. All chemical shifts in nmr experiments are reported as ppm downfield from TMS. The following calibrations were used: $\mathrm{CDCl}_{3} \square=7.26$ and 77.00 ppm, $\mathrm{C}_{6} \mathrm{D}_{6} \square=7.16$ and $128.00 \mathrm{ppm}$. MS and HRMS experiments were performed on a Kratos MS 50 within the service centers at the Kekulé-Department, Bonn.

\section{Synthesis of starting materials}

All $N$-tosylated urea-derivates were prepared from the corresponding nitriles. These were synthesized and reduced using a literature procedure [Y. Tamaru, M. Hojo, H. Higashimura, Z.-I. Yoshida, J. Am. Chem. Soc. 1988, 110, 3994]. The free amine was then directly used without prior purification and reacted with p-toluolsulfonylisocyanate (or phenylsulfonylisocyanate) to give the urea starting materials as white solids:

1) LDA

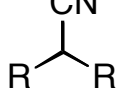

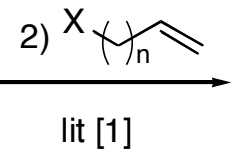<smiles>[R]C([R])(C#N)CC=C</smiles><smiles>[134H]</smiles><smiles>[R]C([R])(C=C)CN</smiles>

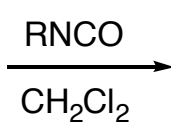<smiles>[R]NC(=O)NCC([R])(P)CC=C</smiles>

\section{Diamination of Alkenes: General Procedures}

\section{General procedure A, preparation of 5-membered diamines:}

To a solution of $\mathrm{Pd}(\mathrm{OAc})_{2}(0.025 \mathrm{mmol}, 11.3 \mathrm{mg})$ and $\mathrm{PhI}(\mathrm{OAc})_{2}(2.00 \mathrm{mmol}, 322 \mathrm{mg})$ in 5 $\mathrm{ml} \mathrm{CH}{ }_{2} \mathrm{Cl}_{2}$ were added the unsaturated urea $(0.50 \mathrm{mmol}), \mathrm{Me}_{4} \mathrm{NCl}(0.50 \mathrm{mmol}, 55 \mathrm{mg})$ and $\mathrm{NaOAc}(0.50 \mathrm{mmol}, 40 \mathrm{mg})$. The mixture was stirred overnight and product formation was followed by tlc where the products appeared as an UV-active spot with a higher retention than the starting compound $\left(\mathrm{R}_{\mathrm{f}}=0.5-0.7\right)$. The reaction was reductively quenched by addition of an aqueous saturated $\mathrm{Na}_{2} \mathrm{~S}_{2} \mathrm{O}_{3}$-solution. The aqueous layer was extracted several times with $\mathrm{CH}_{2} \mathrm{Cl}_{2}$ and the organic layers were extracted with brine. The combined organic layers were 
dried over $\mathrm{MgSO}_{4}$. The solvent was removed under reduced pressure and the product purified by flash-chromatography (hexanes/ $\mathrm{CH}_{2} \mathrm{Cl}_{2} /$ diethylether, $3 / 3 / 1, \mathrm{v} / \mathrm{v}$ ).

\section{General procedure B, catalytic preparation of 6-membered diamines:}

Preparation and workup were the same as in procedure A employing $25 \mathrm{~mol} \%$ of $\mathrm{Pd}(\mathrm{OAc})_{2}$ and no base. Stirring was continued for about 3 days to complete the conversion of all starting material and product formation was followed by tlc where the products appeared as an UVactive spot with a higher retention than the starting compound $\left(\mathrm{R}_{\mathrm{f}}=0.5-0.6\right)$. The reaction was worked up as described and the product was purified by flash-chromatography (hexanes $/ \mathrm{CH}_{2} \mathrm{Cl}_{2} /$ diethylether, $3 / 3 / 1, \mathrm{v} / \mathrm{v}$ ).

\section{Procedure C:}

Preparation and workup were the same as in procedure A employing $10 \mathrm{~mol} \%$ of $\mathrm{Pd}(\mathrm{OAc})_{2}$ and no base. Stirring was continued for 12 hours to complete the conversion of all starting material and product formation was followed by tlc where the product appeared as an UVactive spot with a higher retention than the starting compound $\left(R_{f}=0.6\right)$. The reaction was worked up as described and the product was purified by flash-chromatography (hexanes $/ \mathrm{CH}_{2} \mathrm{Cl}_{2}$ /diethylether, $3 / 3 / 1$, v/v).

\section{Hexahydro-6,6-dimethyl-2-(p-tolylsulfonyl)pyrrolo-[1,2-e]imidazol-3-one}

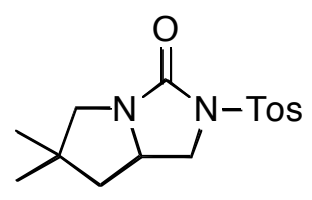

Synthesized according to procedure A.

${ }^{1} \mathrm{H} \mathrm{nmr}\left(300 \mathrm{MHz}, \mathrm{CDCl}_{3}\right): \square=0.96$ (s, 3H), 1.05 (s, 3H), $1.27(\mathrm{dd}, J=9.2,12.4 \mathrm{~Hz}, 1 \mathrm{H})$, $1.78(\mathrm{dd}, J=6.1,12.4 \mathrm{~Hz}, 1 \mathrm{H}), 2.40$ (s, 3H), 3.76 (d, $J=11.3 \mathrm{~Hz}, 1 \mathrm{H}), 3.28$ (d, $J=11.3 \mathrm{~Hz}$, $1 \mathrm{H}), 3.63(\mathrm{dd}, J=4.2,9.2 \mathrm{~Hz}, 1 \mathrm{H}), 3.92(\mathrm{~m}, 1 \mathrm{H}), 4.03(\mathrm{dd}, J=8.7,9.3 \mathrm{~Hz}, 1 \mathrm{H}), 7.30(\mathrm{~d}, J=$ $8.4 \mathrm{~Hz}, 2 \mathrm{H}), 7.89$ (d, $J=8.4 \mathrm{~Hz}, 2 \mathrm{H})$.

${ }^{13} \mathrm{C} \mathrm{nmr}\left(75 \mathrm{MHz}, \mathrm{CDCl}_{3}\right)$ : $\square=21.52,27.53,28.08,40.62,46.02,48.19,54.69,58.41,127.89$, 129.51, 134.97, 144.65, 156.16.

MS: $293.2\left[\mathrm{M}-\mathrm{CH}_{3}\right]^{+}, 252.1$ (18), 244.2 (100), 229.2 (8), 188.1 (25), 155.1 (16), 153.2 (32), 133.1 (3), 105.1 (4), 97.1 (36), 91.1 (38), 82.1 (3), 65.1 (8), 55.1 (58).

HRMS: calc. for $\mathrm{C}_{14} \mathrm{H}_{17} \mathrm{~N}_{2} \mathrm{O}_{3} \mathrm{~S}\left(\mathrm{M}-\mathrm{CH}_{3}\right)$ : 293.0960, found: 293.0962 . 


\section{Hexahydro-6,6-dimethyl-2-phenylsulfonylpyrrolo-[1,2-e]imidazol-3-one}<smiles>CC1(C)CC2CN(S(=O)(=O)Oc3ccccc3)C(=O)N2C1</smiles>

Synthesized according to procedure A.

${ }^{1} \mathrm{H}$ nmr $\left(300 \mathrm{MHz}, \mathrm{CDCl}_{3}\right): \square=0.96$ (s, 3H), 1.06 (s, 3H), $1.28(\mathrm{dd}, J=9.0,12.4 \mathrm{~Hz}, 1 \mathrm{H})$, $1.79(\mathrm{dd}, J=6.3,12.4 \mathrm{~Hz}, 1 \mathrm{H}), 2.77(\mathrm{~d}, J=11.3 \mathrm{~Hz}, 1 \mathrm{H}), 3.29$ (d, $J=11.3 \mathrm{~Hz}, 1 \mathrm{H}), 3.64$ (dd, $J=4.3 .9 .3 \mathrm{~Hz}, 1 \mathrm{H}), 3.94(\mathrm{~m}, 1 \mathrm{H}), 4.04(\mathrm{dd}, J=8.7,9.3 \mathrm{~Hz}, 1 \mathrm{H}), 7.52(\mathrm{~m}, 2 \mathrm{H}), 7.62(\mathrm{tt}, J=$ $1.3,7.4 \mathrm{~Hz}, J=1.3 \mathrm{~Hz}, 1 \mathrm{H}), 8.03(\mathrm{~m}, 2 \mathrm{H})$.

${ }^{13} \mathrm{C} \mathrm{nmr}\left(75 \mathrm{MHz}, \mathrm{CDCl}_{3}\right): \square=27.56,28.11,40.69,46.09,48.33,54.76,48.45,127.92$, $128.96,133.68,137.93,156.09$.

MS: $279.2\left[\mathrm{M}-\mathrm{CH}_{3}\right]^{+}, 238.1$ (40), 230.2 (84), 215.2 (8), 202.2 (4), 174.1 (28), 153.2 (40), 141.1 (12), 97.1 (30), 82.1 (8), 77.1 (42), 55.1 (100).

HRMS: calc. for $\mathrm{C}_{13} \mathrm{H}_{15} \mathrm{~N}_{2} \mathrm{O}_{3} \mathrm{~S}\left(\mathrm{M}-\mathrm{CH}_{3}\right)$ : 279.0803, found: 279.0803 .

\section{Hexahydro-2-(p-tolylsulfonyl)-6,6-diphenylpyrrolo-[1,2-e]imidazol-3-one}

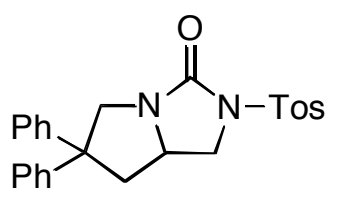

Synthesized according to procedure A.

${ }^{1} \mathrm{H} \mathrm{nmr} \mathrm{(300} \mathrm{MHz,} \mathrm{CDCl}_{3}$, TMS): $\square=2.15$ (dd, $\left.J=9.6,12.0 \mathrm{~Hz}, 1 \mathrm{H}\right), 2.34$ (s, 3H), 2.47 (dd, $J=5.2,12.0 \mathrm{~Hz}, 1 \mathrm{H}), 3.53(\mathrm{dd}, J=5.3,9.3 \mathrm{~Hz}, 1 \mathrm{H}), 3.77(\mathrm{~d}, J=11.7 \mathrm{~Hz}, 1 \mathrm{H}), 3.81(\mathrm{~m}, 1 \mathrm{H})$, $3.94(\mathrm{~d}, J=12.0 \mathrm{~Hz}, 1 \mathrm{H}), 3.97(\mathrm{dd}, J=8.7,9.4 \mathrm{~Hz}, 1 \mathrm{H}), 6.98-7.17(\mathrm{~m}, 10 \mathrm{H}), 7.23(\mathrm{~d}, J=8.2$ $\mathrm{Hz}, 2 \mathrm{H}), 7.80$ (d, $J=8.2 \mathrm{~Hz}, 2 \mathrm{H})$.

${ }^{13} \mathrm{C} \mathrm{nmr}\left(75 \mathrm{MHz}, \mathrm{CDCl}_{3}\right): \square=21.54,43.60,47.99,54.00,56.06,56.78,126.41,126.65$, 127.94, 128.47, 129.58, 134.94, 144.68, 145.16, 145.37, 156.14 .

MS: $432.2\left[\mathrm{M}^{+}\right.$(100), 368.2 (2), 277.2 (35), 252.1 (40), 205.1 (4), 193.1 (10), 179.1 (8), 115.1 (5), 97.1 (35), 91.1 (18), 65.1 (2), 55.1 (32).

HRMS: calc. for $\mathrm{C}_{25} \mathrm{H}_{24} \mathrm{~N}_{2} \mathrm{O}_{3} \mathrm{~S}: 432.1508$, found: 432.1508 . 


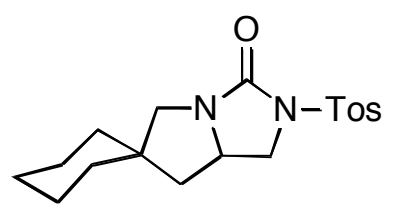

Synthesized according to procedure A.

${ }^{1} \mathrm{H}$ nmr (300 MHz, $\left.\mathrm{CDCl}_{3}\right): \square=1.15-1.50(\mathrm{~m}, 11 \mathrm{H}), 1.89$ (dd, $\left.J=6.4,12.6 \mathrm{~Hz}, 1 \mathrm{H}\right), 2.42$ (s, $3 \mathrm{H}), 2.77(\mathrm{~d}, J=11.7 \mathrm{~Hz}, 1 \mathrm{H}), 3.39$ (d, $J=11.7 \mathrm{~Hz}, 2 \mathrm{H}), 3.64(\mathrm{dd}, J=3.8,9.6 \mathrm{~Hz}, 1 \mathrm{H}), 3.85$ (m, 1H), $3.99(\mathrm{dd}, J=8.4,9.6 \mathrm{~Hz}, 1 \mathrm{H}), 7.31(\mathrm{~d}, J=8.3 \mathrm{~Hz}, 2 \mathrm{H}), 7.90(\mathrm{~d}, J=8.3 \mathrm{~Hz}, 2 \mathrm{H})$.

${ }^{13} \mathrm{C}$ nmr $\left(75 \mathrm{MHz} \mathrm{CDCl}_{3}\right)$ : $\mathrm{\square}=21.59,23.15,23.67,25.49,36.28,37.72,43.92,44.74,48.08$, $54.16,56.41,127.97,129.55,135.02,144.68,156.28$.

MS: $347.2[\mathrm{M}]^{+}, 332.2(2), 298.3$ (1), 284.3 (100), 253.1 (25), 227.2 (1), 193.2 (24), 175.1 (3), 155.1 (20), 138.2 (9), 109,1 (2), 105.1 (4), 97.1 (24), 91.1 (30), 65.1 (4), 55.1 (30).

HRMS: calc. for $\mathrm{C}_{18} \mathrm{H}_{24} \mathrm{~N}_{2} \mathrm{O}_{3} \mathrm{~S}$ (M-H): 347.1429, found: 347.1426 .

\section{Hexahydro-2-(p-tolylsulfonyl)imidazo[1,5-a]pyridine-3(5H)-one}<smiles>O=C1N([18O])CC2CCCCN12</smiles>

Synthesized according to procedure B.

${ }^{1} \mathrm{H} \mathrm{nmr}\left(300 \mathrm{MHz}, \mathrm{CDCl}_{3}\right): \square=1.15-1.43(\mathrm{~m}, 3 \mathrm{H}), 1.62(\mathrm{~m}, 1 \mathrm{H}), 1.83(\mathrm{~m}, 2 \mathrm{H}), 2.42(\mathrm{~s}, 3 \mathrm{H})$, $2.64(\mathrm{td}, J=3.6,13.2 \mathrm{~Hz}, 1 \mathrm{H}), 3.35-3.49(\mathrm{~m}, 2 \mathrm{H}), 3.80(\mathrm{dd}, J=4.2,13.6,1 \mathrm{H}), 3.95(\mathrm{dd}, J=$ 7.8, 8.7 Hz, 1H), 7.32 (d, $J=8.3 \mathrm{~Hz}, 2 \mathrm{H}), 7.90$ (d, $J=8.3 \mathrm{~Hz}, 2 \mathrm{H})$.

${ }^{13} \mathrm{C} \mathrm{nmr}\left(75 \mathrm{MHz}, \mathrm{CDCl}_{3}\right): \square=21.58,22.77,24.00,30.66,41.97,48.45,51.87,128.01$, $129.55,135.15,144.55,152.65$.

MS: $293.1[\mathrm{M}]^{+}, 244.2$ (4), 230.2 (100), 215.2 (8), 187.1 (6), 174.1 (4), 155.1 (12), 139.1 (50), 119.1 (8), 97.1 (5), 91.1 (43), 82.1 (4), 65.1 (8), 55.1 (10).

HRMS: calc. for $\mathrm{C}_{14} \mathrm{H}_{17} \mathrm{~N}_{2} \mathrm{O}_{3} \mathrm{~S}$ (M-H): 293.0959, found: 293.0962 . 


\section{Hexahydro-6,6-dimethyl-2-(p-tolylsulfonyl)imidazo[1,5-a]pyridine-3(5H)-one}

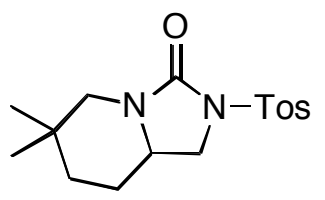

Synthesized according to procedure B.

${ }^{1} \mathrm{H} \mathrm{nmr}\left(300 \mathrm{MHz}, \mathrm{CDCl}_{3}\right): \square=0.82(\mathrm{~s}, 3 \mathrm{H}), 0.91(\mathrm{~s}, 3 \mathrm{H}), 1.28(\mathrm{td}, J=3.7,14.0 \mathrm{~Hz}, 1 \mathrm{H}), 1.47$ $(\mathrm{m}, 2 \mathrm{H}), 1.66(\mathrm{~m}, 1 \mathrm{H}), 2.40(\mathrm{~d}, J=11.5 \mathrm{~Hz}, 1 \mathrm{H}), 2.41(\mathrm{~s}, 3 \mathrm{H}), 3.39-3.47(\mathrm{~m}, 3 \mathrm{H}), 3.95(\mathrm{dd}, J$ $=8.1,9.0 \mathrm{~Hz}, 1 \mathrm{H}), 7.30(\mathrm{~d}, J=8.5 \mathrm{~Hz}, 2 \mathrm{H}), 7.90(\mathrm{~d}, J=8.5 \mathrm{~Hz}, 2 \mathrm{H})$.

${ }^{13} \mathrm{C} \mathrm{nmr}\left(75 \mathrm{MHz}, \mathrm{CDCl}_{3}\right): \square=21.58,23.10,27.10,28.59,30.27,36.34,48.23,51.54,51.75$, $128.00,129.54,135.15,144.53,152.94$.

MS: $321.1[\mathrm{M}]^{+}, 307.1$ (5), 258.2 (100), 243.2 (25), 239.1 (2), 189.1 (38), 167.1 (28), 155.0 (32), $119.1(2), 112.1(8), 91.0(30), 69.1(3), 55.1(6)$.

HRMS: calc. for $\mathrm{C}_{16} \mathrm{H}_{21} \mathrm{~N}_{2} \mathrm{O}_{3} \mathrm{~S}(\mathrm{M}-\mathrm{H}): 321.1272$, found: 321.1269 .

\section{Spiro[cyclohexan-1',6-hexahydro-2-(p-tolylsulfonyl)imidazo[1,5-a]pyridine-3(5H)-one]}

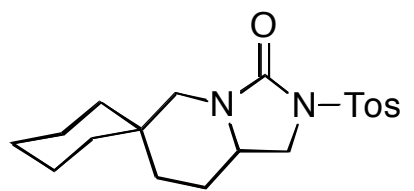

Synthesized according to procedure B.

${ }^{1} \mathrm{H}$ nmr (300 MHz, $\left.\mathrm{CDCl}_{3}\right): \square=1.09-1.70(\mathrm{~m}, 14 \mathrm{H}), 2.26(\mathrm{~d}, J=13.4 \mathrm{~Hz}, 1 \mathrm{H}), 2.41(\mathrm{~s}, 3 \mathrm{H})$, $3.37(\mathrm{~m}, 1 \mathrm{H}), 3.42(\mathrm{dd}, J=5.3,9.4 \mathrm{~Hz}, 1 \mathrm{H}), 3.79(\mathrm{dd}, J=2.2,13.2 \mathrm{~Hz}, 1 \mathrm{H}), 3.88(\mathrm{dd}, J=8.7$, $9.2 \mathrm{~Hz}, 1 \mathrm{H}), 7.30(\mathrm{~d}, J=8.2 \mathrm{~Hz}, 2 \mathrm{H}), 7.90(\mathrm{~d}, J=8.2 \mathrm{~Hz}, 2 \mathrm{H})$.

${ }^{13} \mathrm{C} \mathrm{nmr}\left(75 \mathrm{MHz}, \mathrm{CDCl}_{3}\right): \square=21.34,21.46,21.57,26.25,26.34,30.63,32.73,34.89,37.96$, $48.15,49.13,51.95,127.99,129.51,135.09,144.51,152.91$.

MS: $362.2[\mathrm{M}]^{+}, 298.3$ (100), 253.1 (20), 207.2 (50), 189.1 (20), 155.1 (40), 139.1 (4), 111.1 (16), 99.1 (28), 91.1 (64), 81.1 (10), 67.1 (12), 55.1 (12).

HRMS: calc. for $\mathrm{C}_{19} \mathrm{H}_{26} \mathrm{~N}_{2} \mathrm{O}_{3} \mathrm{~S}: 362.1664$, found: 362.1662 . 


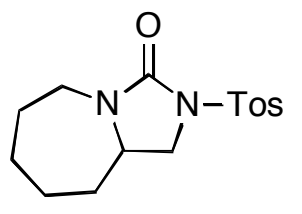

Synthesized according to procedure C.

${ }^{1} \mathrm{H}$ nmr (300 MHz, $\left.\mathrm{CDCl}_{3}\right): \square=1.16-1.60(\mathrm{~m}, 6 \mathrm{H}), 1.63-1.72(\mathrm{~m}, 1 \mathrm{H}), 1.91-2.02(\mathrm{~m}, 1 \mathrm{H}), 2.43$ (s, 3H), $3.21(\mathrm{~m}, 2 \mathrm{H}), 3.65(\mathrm{dd}, J=7.5,11.2 \mathrm{~Hz}, 1 \mathrm{H}), 3.74(\mathrm{dd}, J=5.1,11.4 \mathrm{~Hz}, 1 \mathrm{H}), 3.99$ (m, $1 \mathrm{H}), 7.31(\mathrm{~d}, J=8.2,2 \mathrm{H}), 7.77(\mathrm{~d}, J=8.2,2 \mathrm{H})$.

${ }^{13} \mathrm{C} \mathrm{nmr}\left(75 \mathrm{MHz}, \mathrm{CDCl}_{3}\right)$ : $\square=21.58,25.39,26.00,29.32,34.82,40.09,48.13,60.90,126.89$, $129.88,136.74,144.75,151.88$

MS: $347.2[\mathrm{M}]^{+}, 332.2(2), 298.3$ (1), 284.3 (100), 253.1 (25), 227.2 (1), 193.2 (24), 175.1 (3), 155.1 (20), 138.2 (9), 109,1 (2), 105.1 (4), 97.1 (24), 91.1 (30), 65.1 (4), 55.1 (30).

\section{Hexahydro-2-(p-tolylsulfonyl)-6,6,8-trimethylpyrrolo-[1,2-e]imidazol-3-one}<smiles>CC1(C)CN2C(=O)N([13CH3])CC2(C)C1</smiles>

Synthesized according to procedure A.

${ }^{1} \mathrm{H} \mathrm{nmr}\left(300 \mathrm{MHz}, \mathrm{CDCl}_{3}\right): \square=0.66(\mathrm{~s}, 3 \mathrm{H}), 1.07$ (s, 3H), 1.29 (s, 3H), 1.59 (d, J=4.9 Hz, 2H), $2.41(\mathrm{~s}, 3 \mathrm{H}), 2.73(\mathrm{~d}, J=12.1 \mathrm{~Hz}, 1 \mathrm{H}), 3.41(\mathrm{~d}, J=11.9 \mathrm{~Hz}, 1 \mathrm{H}), 3.69$ (d, $J=1.0 \mathrm{~Hz}$, $2 \mathrm{H}), 7.30(\mathrm{~d}, J=8.2 \mathrm{~Hz}, 2 \mathrm{H}), 7.91(\mathrm{~d}, J=8.2 \mathrm{~Hz}, 2 \mathrm{H})$.

${ }^{13} \mathrm{C} \mathrm{nmr}\left(75 \mathrm{MHz}, \mathrm{CDCl}_{3}\right)$ : $\square=21.55,27.48,27.69,28.42,41.08,52.90,56.80,57.48,62.16$, $128.02,129.47,134.75,144.66,156.19$.

MS: $321.2[\mathrm{M}]^{+}, 307.2(61), 266.1$ (82), 258.2 (96), 202.2 (14), 167.2 (42), 155.1 (39), 111.1 (59), 01.1 (77), 69.1 (100).

HRMS: calc. for $\mathrm{C}_{16} \mathrm{H}_{21} \mathrm{~N}_{2} \mathrm{O}_{3} \mathrm{~S}$ [M-H]: 321.1272. Found: 321.1276. 


\section{Tricyclic urea 2a}<smiles>O=C1N([18O])CC2Cc3ccccc3N12</smiles>

Synthesized according to procedure A.

${ }^{1} \mathrm{H}$ nmr $\left(500 \mathrm{MHz}, \mathrm{CDCl}_{3}\right): \square=2.42(\mathrm{~s}, 3 \mathrm{H}), 2.92(\mathrm{dd}, J=9.6,15.8 \mathrm{~Hz}, 1 \mathrm{H}), 3.27(\mathrm{dd}, J=$ 8.7, $15.8 \mathrm{~Hz}, 1 \mathrm{H}$ ), 3.65 (ps-t, $J=9.0 \mathrm{~Hz}, 1 \mathrm{H}$ ), 4.45 (dd, $J=8.4,9.3 \mathrm{~Hz}, 1 \mathrm{H}$ ), 4.66 (pseudoquin, $J=8.7 \mathrm{~Hz}, 1 \mathrm{H}), 7.04(\mathrm{td}, J=1.0,7.5 \mathrm{~Hz}, 1 \mathrm{H}), 7.16(\mathrm{~d}, J=8.2 \mathrm{~Hz}, 1 \mathrm{H}) 7.18(\mathrm{~d}, J=1.0$, $7.7 \mathrm{~Hz}, 1 \mathrm{H}), 7.31$ (d, $J=8.4 \mathrm{~Hz}, 2 \mathrm{H}), 7.40$ (d, $J=7.8 \mathrm{~Hz}, 1 \mathrm{H}), 7.96(\mathrm{~d}, J=8.4 \mathrm{~Hz}, 2 \mathrm{H})$.

${ }^{13} \mathrm{C} \mathrm{nmr}\left(125 \mathrm{MHz}, \mathrm{CDCl}_{3}\right)$ : $\square=21.63,35.78,51.19,55.67,115.17,124.56,125.16,128.14$, $128.27,129.73,132.04,134.81,140.12,145.01,151.22$.

MS: $328.1[\mathrm{M}]^{+}$(100), 272.1 (35), 264.2 (10), 173.1 (16), 155.0 (32), 130.1 (56), 117.1 (16), 91.1 (48), 84.0 (12), 65.1 (10), 56.1 (15).

HRMS: calc. for $\mathrm{C}_{17} \mathrm{H}_{16} \mathrm{~N}_{2} \mathrm{O}_{3} \mathrm{~S}$ : 328.0882 , found: 328.0877 .

\section{Tricyclic urea 2b}<smiles>CC(=O)Oc1ccc2c(c1)CC1CN([18O])C(=O)N21</smiles>

Obtained as side-product from a reaction according to procedure $\mathrm{A}$ in the absence of base.

${ }^{1} \mathrm{H} \mathrm{nmr}\left(300 \mathrm{MHz}, \mathrm{CDCl}_{3}\right): \square=2.26(\mathrm{~s}, 3 \mathrm{H}), 2.42(\mathrm{~s}, 3 \mathrm{H}), 2.93(\mathrm{dd}, J=9.5,16.0 \mathrm{~Hz}, 1 \mathrm{H})$, $3.26(\mathrm{dd}, J=8.8,16.0 \mathrm{~Hz}, 1 \mathrm{H}), 3.64$ (pseudo-t, $J=9.0 \mathrm{~Hz}, 1 \mathrm{H}), 4.44$ (dd, $J=8.5,9.2 \mathrm{~Hz}$, $1 \mathrm{H}), 4.68$ (pseudo-quin, $J=8.8 \mathrm{~Hz}, 1 \mathrm{H}), 6.90(\mathrm{~m}, 2 \mathrm{H}), 7.32(\mathrm{~d}, J=8.0 \mathrm{~Hz}, 2 \mathrm{H}), 7.37$ (d, $J=$ $8.3 \mathrm{~Hz}, 1 \mathrm{H}), 7.94(\mathrm{~d}, J=8.3 \mathrm{~Hz}, 2 \mathrm{H})$.

${ }^{13} \mathrm{C} \mathrm{nmr}\left(75 \mathrm{MHz}, \mathrm{CDCl}_{3}\right)$ : $\square=20.97,21.62,35.86,51.12,55.92,115.56,118.97,121.14$, $128.24,129.78,133.34,134.59,138.02,145.15,147.49,151.38,169.75$ 
MS: $386.1[\mathrm{M}]^{+}, 344.1$ (100), 328.1 (6), 189.1 (25), 155.0 (16), 146.1 (32), 133.0 (12), 130.1 (8), $91.1(40), 65.1(5)$.

HRMS: calc. for $\mathrm{C}_{19} \mathrm{H}_{18} \mathrm{~N}_{2} \mathrm{O}_{3} \mathrm{~S}: 386.0936$, found: 386.0940 . 


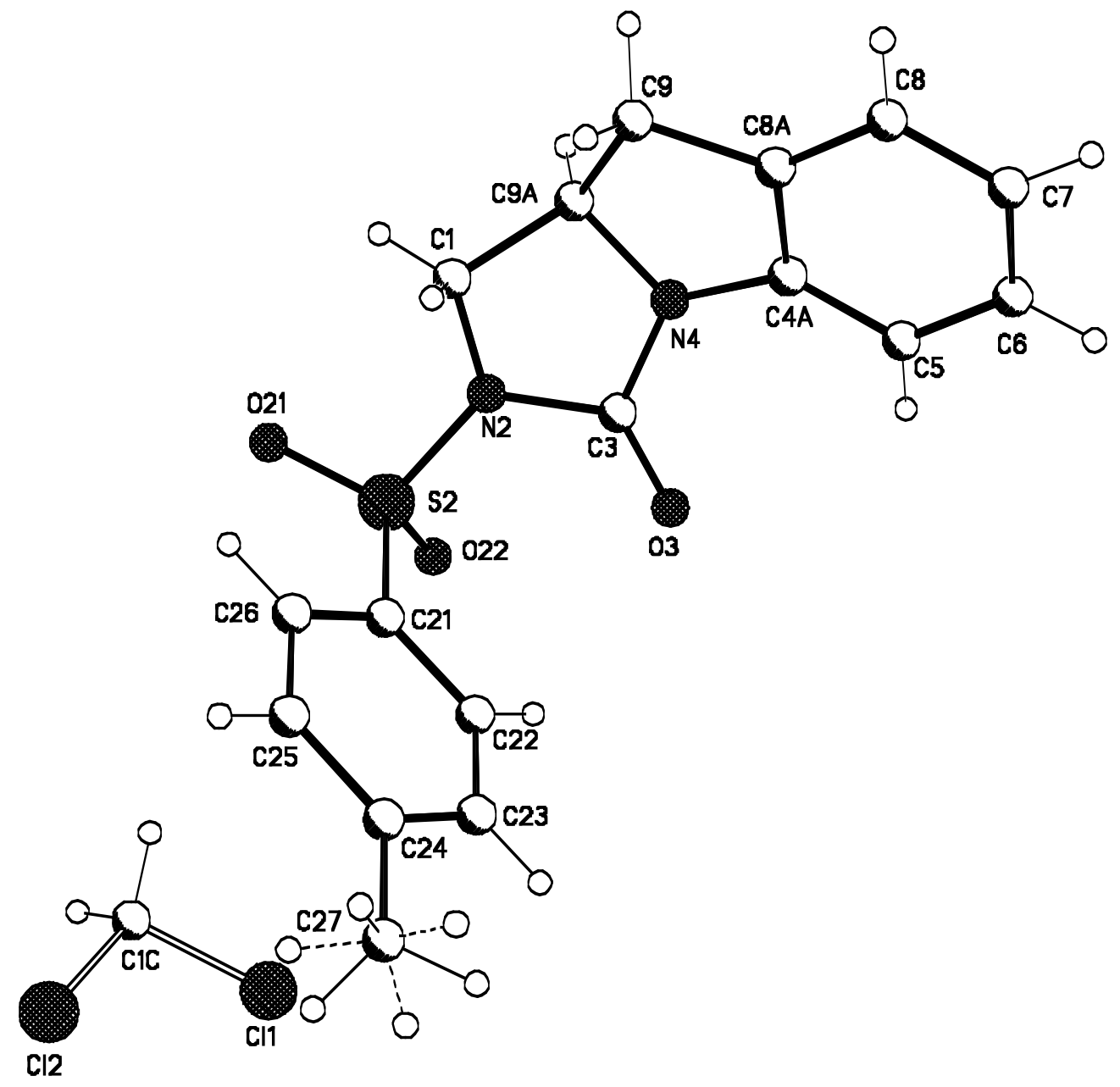

Table 1. Crystal data and structure refinement for $\mathbf{2 a}$.

Empirical formula

Formula weight

Temperature

Wavelength

Crystal system, space group

Unit cell dimensions
$\mathrm{C} 17.50 \mathrm{H} 17 \mathrm{Cl}$ N2 $\mathrm{O} 3 \mathrm{~S}$

$\mathrm{C} 17 \mathrm{H} 16 \mathrm{~N} 2 \mathrm{O} 3 \mathrm{~S}-0.5 \mathrm{CH} 2 \mathrm{Cl} 2$

370.84

$123(2) \mathrm{K}$

$0.71073 \mathrm{~A}$

Triclinic, P-1 (No.2)

$\mathrm{a}=5.9460(3) \mathrm{A} \quad$ alpha $=96.432(3) \mathrm{deg} \cdot$

$\mathrm{b}=11.5541(7) \mathrm{A}$ beta $=95.564(4) \mathrm{deg}$.

$\mathrm{C}=12.6351(9) \mathrm{A}$ gamma $=103.692(2) \mathrm{deg}$. 


\begin{tabular}{|c|c|}
\hline Volume & $831.18(9) \quad A^{\wedge} 3$ \\
\hline Z, Calculated density & $2, \quad 1.482 \mathrm{Mg} / \mathrm{m}^{\wedge} 3$ \\
\hline Absorption coefficient & $0.375 \mathrm{~mm}^{\wedge}-1$ \\
\hline $\mathrm{F}(000)$ & 386 \\
\hline Crystal size & $0.50 \times 0.40 \times 0.05 \mathrm{~mm}$ \\
\hline Diffractometer & Nonius KappaCCD \\
\hline Theta range for data collection & 3.52 to $25.02 \mathrm{deg}$ \\
\hline Limiting indices & $-6<=\mathrm{h}<=7, \quad-13<=\mathrm{k}<=13,-14<=1<=14$ \\
\hline Reflections collected / unique & $6439 / 2898[R($ int $)=0.0384]$ \\
\hline Completeness to theta $=25.02$ & $99.2 \%$ \\
\hline Absorption correction & None \\
\hline Refinement method & Full-matrix least-squares on $\mathrm{F}^{\wedge} 2$ \\
\hline Data / restraints / parameters & $2898 / 21 / 231$ \\
\hline Goodness-of-fit on $\mathrm{F}^{\wedge} 2$ & 1.103 \\
\hline Final R indices [I>2sigma(I)] & $\mathrm{R} 1=0.0644, \mathrm{WR} 2=0.1822$ \\
\hline $\mathrm{R}$ indices (all data) & $\mathrm{R} 1=0.0855, \mathrm{wR} 2=0.1948$ \\
\hline Largest diff. peak and hole & 0.651 and -1.036 e.A^-3 \\
\hline
\end{tabular}


Table 2. Atomic coordinates $\left(\mathrm{x}^{\left.10^{\wedge} 4\right)}\right.$ and equivalent isotropic displacement parameters $\left(A^{\wedge} 2 \times 10^{\wedge} 3\right)$ for 2 a.

$\mathrm{U}(\mathrm{eq})$ is defined as one third of the trace of the orthogonalized Uij tensor.

\begin{tabular}{|c|c|c|c|c|c|}
\hline & $\mathrm{x}$ & $\mathrm{y}$ & $\mathrm{z}$ & $\mathrm{U}(\mathrm{eq})$ & \\
\hline$C(1)$ & $3232(7)$ & $3880(3)$ & $758(3)$ & $30(1)$ & \\
\hline $\mathrm{N}(2)$ & $1365(5)$ & $4333(3)$ & $1238(2)$ & $29(1)$ & \\
\hline $\mathrm{S}(2)$ & $1571(2)$ & $5773(1)$ & $1604(1)$ & $29(1)$ & \\
\hline$O(21)$ & $3121(5)$ & $6389(2)$ & $937(2)$ & $35(1)$ & \\
\hline $0(22)$ & $-756(5)$ & $5918(2)$ & $1588(2)$ & $34(1)$ & \\
\hline$C(21)$ & $2935(7)$ & $6125(3)$ & $2945(3)$ & $30(1)$ & \\
\hline$C(22)$ & $1569(7)$ & $5942(4)$ & $3767(3)$ & $38(1)$ & \\
\hline$C(23)$ & $2649(7)$ & $6219(4)$ & $4826(3)$ & $41(1)$ & \\
\hline$C(24)$ & $5047(7)$ & $6681(4)$ & $5057(3)$ & $38(1)$ & \\
\hline$C(25)$ & $6349(8)$ & $6862(4)$ & $4215(3)$ & $47(1)$ & \\
\hline$C(26)$ & $5315(7)$ & $6581(4)$ & $3152(3)$ & $38(1)$ & \\
\hline$C(27)$ & $6188(8)$ & $6982(5)$ & $6205(3)$ & $55(1)$ & \\
\hline$C(3)$ & $-173(6)$ & $3395(3)$ & $1648(3)$ & $29(1)$ & \\
\hline$O(3)$ & $-1502(4)$ & $3518(2)$ & $2296(2)$ & $35(1)$ & \\
\hline $\mathrm{N}(4)$ & $181(5)$ & $2354(3)$ & $1155(2)$ & $30(1)$ & \\
\hline$C(4 A)$ & $20(7)$ & $1254(3)$ & $1573(3)$ & $31(1)$ & \\
\hline$C(5)$ & $-1601(7)$ & $711(3)$ & $2192(3)$ & $38(1)$ & \\
\hline$C(6)$ & $-1379(8)$ & $-388(4)$ & $2498(4)$ & $46(1)$ & \\
\hline$C(7)$ & $356(8)$ & $-902(4)$ & $2178(4)$ & $47(1)$ & \\
\hline$C(8)$ & $1974(8)$ & $-339(4)$ & $1560(3)$ & $41(1)$ & \\
\hline$C(8 A)$ & $1794(7)$ & $754(3)$ & $1251(3)$ & $32(1)$ & \\
\hline$C(9)$ & $3347(7)$ & $1587(3)$ & $624(3)$ & $37(1)$ & \\
\hline$C(9 A)$ & $2010(7)$ & $2554(3)$ & $437(3)$ & $33(1)$ & \\
\hline$C(1 C)$ & $4290(20)$ & $9730(20)$ & $4344(10)$ & $141(7)$ & s.o.f. $=0.50$ \\
\hline $\mathrm{Cl}(1)$ & $1892(9)$ & $9173(7)$ & $4948(4)$ & $160(2)$ & s.o.f. $=0.50$ \\
\hline $\mathrm{Cl}(2)$ & $6661(9)$ & $10367(7)$ & $5285(5)$ & $153(2)$ & s.o.f. $=0.50$ \\
\hline
\end{tabular}


Table 3. Bond lengths [A] and angles [deg] for 2a.

\begin{tabular}{|c|c|}
\hline $\mathrm{C}(1)-\mathrm{N}(2)$ & $1.486(4)$ \\
\hline$C(1)-C(9 A)$ & $1.522(5)$ \\
\hline$N(2)-C(3)$ & $1.424(5)$ \\
\hline$N(2)-S(2)$ & $1.648(3)$ \\
\hline$S(2)-O(21)$ & $1.428(3)$ \\
\hline$S(2)-O(22)$ & $1.431(3)$ \\
\hline$S(2)-C(21)$ & $1.766(4)$ \\
\hline$C(21)-C(26)$ & $1.376(6)$ \\
\hline$C(21)-C(22)$ & $1.384(5)$ \\
\hline$C(22)-C(23)$ & $1.394(5)$ \\
\hline$C(23)-C(24)$ & $1.389(6)$ \\
\hline$C(24)-C(25)$ & $1.381(6)$ \\
\hline$C(24)-C(27)$ & $1.507(5)$ \\
\hline$C(25)-C(26)$ & $1.391(6)$ \\
\hline $\mathrm{C}(3)-\mathrm{O}(3)$ & $1.212(4)$ \\
\hline $\mathrm{C}(3)-\mathrm{N}(4)$ & $1.363(5)$ \\
\hline $\mathrm{N}(4)-\mathrm{C}(4 \mathrm{~A})$ & $1.417(4)$ \\
\hline$N(4)-C(9 A)$ & $1.475(5)$ \\
\hline$C(4 A)-C(5)$ & $1.380(5)$ \\
\hline$C(4 A)-C(8 A)$ & $1.390(5)$ \\
\hline$C(5)-C(6)$ & $1.400(5)$ \\
\hline$C(6)-C(7)$ & $1.378(6)$ \\
\hline$C(7)-C(8)$ & $1.387(6)$ \\
\hline$C(8)-C(8 A)$ & $1.387(5)$ \\
\hline$C(8 A)-C(9)$ & $1.516(5)$ \\
\hline$C(9)-C(9 A)$ & $1.543(5)$ \\
\hline$C(1 C)-C l(1)$ & $1.709(10)$ \\
\hline$C(1 C)-C l(2)$ & $1.711(10)$ \\
\hline $\mathrm{N}(2)-\mathrm{C}(1)-\mathrm{C}(9 \mathrm{~A})$ & $100.9(3)$ \\
\hline$C(3)-N(2)-C(1)$ & $110.6(3)$ \\
\hline $\mathrm{C}(3)-\mathrm{N}(2)-\mathrm{S}(2)$ & $123.3(2)$ \\
\hline $\mathrm{C}(1)-\mathrm{N}(2)-\mathrm{S}(2)$ & $123.2(2)$ \\
\hline $\mathrm{O}(21)-\mathrm{S}(2)-\mathrm{O}(22)$ & $120.48(15)$ \\
\hline $\mathrm{O}(21)-\mathrm{S}(2)-\mathrm{N}(2)$ & $105.05(15)$ \\
\hline $\mathrm{O}(22)-\mathrm{S}(2)-\mathrm{N}(2)$ & $107.18(16)$ \\
\hline $\mathrm{O}(21)-\mathrm{S}(2)-\mathrm{C}(21)$ & $108.45(17)$ \\
\hline $\mathrm{O}(22)-\mathrm{S}(2)-\mathrm{C}(21)$ & $108.21(17)$ \\
\hline $\mathrm{N}(2)-\mathrm{S}(2)-\mathrm{C}(21)$ & $106.67(15)$ \\
\hline$C(26)-C(21)-C(22)$ & $121.5(4)$ \\
\hline$C(26)-C(21)-S(2)$ & $119.7(3)$ \\
\hline$C(22)-C(21)-S(2)$ & $118.9(3)$ \\
\hline$C(21)-C(22)-C(23)$ & $118.8(4)$ \\
\hline$C(24)-C(23)-C(22)$ & $120.9(4)$ \\
\hline$C(25)-C(24)-C(23)$ & $118.6(4)$ \\
\hline$C(25)-C(24)-C(27)$ & $121.1(4)$ \\
\hline$C(23)-C(24)-C(27)$ & $120.3(4)$ \\
\hline$C(24)-C(25)-C(26)$ & $121.6(4)$ \\
\hline$C(21)-C(26)-C(25)$ & $118.6(4)$ \\
\hline $\mathrm{O}(3)-\mathrm{C}(3)-\mathrm{N}(4)$ & $128.3(3)$ \\
\hline $\mathrm{O}(3)-\mathrm{C}(3)-\mathrm{N}(2)$ & $126.5(3)$ \\
\hline $\mathrm{N}(4)-\mathrm{C}(3)-\mathrm{N}(2)$ & $105.2(3)$ \\
\hline $\mathrm{C}(3)-\mathrm{N}(4)-\mathrm{C}(4 \mathrm{~A})$ & $127.8(3)$ \\
\hline $\mathrm{C}(3)-\mathrm{N}(4)-\mathrm{C}(9 \mathrm{~A})$ & $113.2(3)$ \\
\hline$C(4 A)-N(4)-C(9 A)$ & $111.1(3)$ \\
\hline$C(5)-C(4 A)-C(8 A)$ & $123.0(3)$ \\
\hline $\mathrm{C}(5)-\mathrm{C}(4 \mathrm{~A})-\mathrm{N}(4)$ & $128.0(4)$ \\
\hline $\mathrm{C}(8 \mathrm{~A})-\mathrm{C}(4 \mathrm{~A})-\mathrm{N}(4)$ & $109.0(3)$ \\
\hline$C(4 A)-C(5)-C(6)$ & $116.6(4)$ \\
\hline$C(7)-C(6)-C(5)$ & $121.2(4)$ \\
\hline
\end{tabular}




$\begin{array}{ll}C(6)-C(7)-C(8) & 121.2(4) \\ C(7)-C(8)-C(8 A) & 118.6(4) \\ C(8)-C(8 A)-C(4 A) & 119.3(4) \\ C(8)-C(8 A)-C(9) & 130.1(4) \\ C(4 A)-C(8 A)-C(9) & 110.5(3) \\ C(8 A)-C(9)-C(9 A) & 103.6(3) \\ N(4)-C(9 A)-C(1) & 103.1(3) \\ N(4)-C(9 A)-C(9) & 104.3(3) \\ C(1)-C(9 A)-C(9) & 120.0(3) \\ C l(1)-C(1 C)-C l(2) & 110.6(8)\end{array}$


Table 4. Anisotropic displacement parameters ( $A^{\wedge} 2 \times 10^{\wedge} 3$ ) for $2 a$. The anisotropic displacement factor exponent takes the form: $-2 \mathrm{pi}^{\wedge} 2\left[\mathrm{~h}^{\wedge} 2 \mathrm{a}{ }^{\wedge} 2 \mathrm{U} 11+\ldots+2 \mathrm{~h} \mathrm{k} \mathrm{a} * \mathrm{~b} * \mathrm{U} 12\right]$

\begin{tabular}{|c|c|c|c|c|c|c|}
\hline & U11 & $\mathrm{U} 22$ & U33 & $\mathrm{U} 23$ & U13 & $\mathrm{U} 12$ \\
\hline$C(1)$ & 27 ( 2 ) & 35 ( 2 ) & 31 ( 2 ) & $8(2)$ & $8(2)$ & $7(2)$ \\
\hline $\mathrm{N}(2)$ & $24(2)$ & 32 ( 2 ) & 31 ( 2 ) & 9 (1) & $7(1)$ & $4(1)$ \\
\hline$S(2)$ & 29 ( 1 ) & 31 ( 1 ) & 28 ( 1 ) & 10 (1) & $5(1)$ & $7(1)$ \\
\hline$O(21)$ & $40(2)$ & 35 (1) & 31 ( 1 ) & 14 ( 1 ) & 12 ( 1 ) & $4(1)$ \\
\hline$O(22)$ & $33(2)$ & 42 ( 2 ) & 32 ( 1 ) & 10 (1) & $4(1)$ & $14(1)$ \\
\hline$C(21)$ & 29 ( 2 ) & $33(2)$ & $30(2)$ & 8 ( 2 ) & $6(2)$ & 7 (2) \\
\hline$C(22)$ & $27(2)$ & $50(2)$ & $34(2)$ & 12 ( 2 ) & $6(2)$ & $2(2)$ \\
\hline$C(23)$ & $34(2)$ & 59 (3) & 29 ( 2 ) & $14(2)$ & $8(2)$ & $6(2)$ \\
\hline$C(24)$ & $34(2)$ & $51(2)$ & 29 (2) & $5(2)$ & $3(2)$ & $11(2)$ \\
\hline$C(25)$ & $25(2)$ & $67(3)$ & $45(2)$ & $2(2)$ & $3(2)$ & $9(2)$ \\
\hline$C(26)$ & $26(2)$ & 49 ( 2 ) & 37 ( 2 ) & $4(2)$ & 9 ( 2 ) & $5(2)$ \\
\hline$C(27)$ & 41 ( 3 ) & $84(3)$ & 36 ( 2 ) & 4 ( 2 ) & $-1(2)$ & $15(2)$ \\
\hline$C(3)$ & $23(2)$ & $34(2)$ & 31 (2) & $10(2)$ & 1 (2) & $4(2)$ \\
\hline$O(3)$ & $26(2)$ & $42(2)$ & $42(2)$ & 15 (1) & $14(1)$ & $7(1)$ \\
\hline $\mathrm{N}(4)$ & 25 (2) & $30(2)$ & $36(2)$ & 12 (1) & $6(1)$ & $3(1)$ \\
\hline$C(4 A)$ & $28(2)$ & $30(2)$ & $32(2)$ & $7(2)$ & $-2(2)$ & $2(2)$ \\
\hline$C(5)$ & $32(2)$ & $38(2)$ & $43(2)$ & $14(2)$ & $6(2)$ & $3(2)$ \\
\hline$C(6)$ & $46(3)$ & $40(2)$ & $51(3)$ & $19(2)$ & $11(2)$ & $1(2)$ \\
\hline$C(7)$ & $56(3)$ & $30(2)$ & $54(3)$ & 15 ( 2) & 4 (2) & $5(2)$ \\
\hline$C(8)$ & $39(2)$ & $33(2)$ & $46(2)$ & $3(2)$ & $-2(2)$ & $8(2)$ \\
\hline$C(8 A)$ & $30(2)$ & $33(2)$ & $30(2)$ & $4(2)$ & $-1(2)$ & $3(2)$ \\
\hline$C(9)$ & $36(2)$ & $38(2)$ & $40(2)$ & $7(2)$ & $9(2)$ & $11(2)$ \\
\hline$C(9 A)$ & 29 (2) & $37(2)$ & $32(2)$ & 8 (2) & 7 (2) & $5(2)$ \\
\hline $\mathrm{Cl}(1)$ & $106(4)$ & $264(7)$ & $84(3)$ & $48(3)$ & $18(3)$ & $-16(4)$ \\
\hline $\mathrm{Cl}(2)$ & $86(4)$ & $233(7)$ & $108(4)$ & $-22(4)$ & $-21(3)$ & 11 ( 4 ) \\
\hline
\end{tabular}


Table 5. Hydrogen coordinates $\left(\mathrm{x}^{10^{\wedge}} 4\right)$ and isotropic displacement parameters $\left(A^{\wedge} 2 \times 10^{\wedge} 3\right)$ for 2 a.

\begin{tabular}{|c|c|c|c|c|c|c|}
\hline & $\mathrm{x}$ & $\mathrm{y}$ & $\mathrm{z}$ & \multicolumn{2}{|c|}{$\mathrm{U}(\mathrm{eq})$} & \\
\hline $\mathrm{H}(1 \mathrm{~A})$ & 4632 & 3995 & 1289 & 36 & & \\
\hline $\mathrm{H}(1 \mathrm{~B})$ & 3682 & 4274 & 127 & 36 & & \\
\hline $\mathrm{H}(22)$ & -74 & 5632 & 3613 & 45 & & \\
\hline $\mathrm{H}(23)$ & 1731 & 6090 & 5396 & 49 & & \\
\hline $\mathrm{H}(25)$ & 7989 & 7186 & 4365 & 56 & & \\
\hline $\mathrm{H}(26)$ & 6234 & 6702 & 2580 & 45 & & \\
\hline $\mathrm{H}(27 \mathrm{~A})$ & 5170 & 6531 & 6664 & 82 & s.o.f.= & 0.50 \\
\hline $\mathrm{H}(27 \mathrm{~B})$ & 6449 & 7846 & 6439 & 82 & s.o.f.= & 0.50 \\
\hline $\mathrm{H}(27 \mathrm{C})$ & 7687 & 6764 & 6260 & 82 & s.o.f.= & 0.50 \\
\hline $\mathrm{H}(27 \mathrm{D})$ & 7701 & 7563 & 6244 & 82 & s.o.f.= & 0.50 \\
\hline $\mathrm{H}(27 \mathrm{E})$ & 6421 & 6248 & 6469 & 82 & s.o.f.= & 0.50 \\
\hline $\mathrm{H}(27 \mathrm{~F})$ & 5184 & 7330 & 6649 & 82 & s.o.f.= & 0.50 \\
\hline $\mathrm{H}(5)$ & -2806 & 1066 & 2401 & 45 & & \\
\hline H ( 6) & -2445 & -787 & 2933 & 55 & & \\
\hline $\mathrm{H}(7)$ & 446 & -1657 & 2386 & 57 & & \\
\hline $\mathrm{H}(8)$ & 3179 & -694 & 1352 & 49 & & \\
\hline $\mathrm{H}(9 \mathrm{~A})$ & 3553 & 1152 & -67 & 44 & & \\
\hline $\mathrm{H}(9 \mathrm{~B})$ & 4900 & 1950 & 1043 & 44 & & \\
\hline $\mathrm{H}(9 \mathrm{~A} 1)$ & 1269 & 2416 & -326 & 39 & & \\
\hline $\mathrm{H}(1 \mathrm{C} 1)$ & 3970 & 10345 & 3902 & 169 & s.o.f.= & 0.50 \\
\hline $\mathrm{H}(1 \mathrm{C} 2)$ & 4631 & 9075 & 3863 & 169 & s.o.f.= & 0.50 \\
\hline
\end{tabular}


Table 6. Torsion angles [deg] for 2 a.

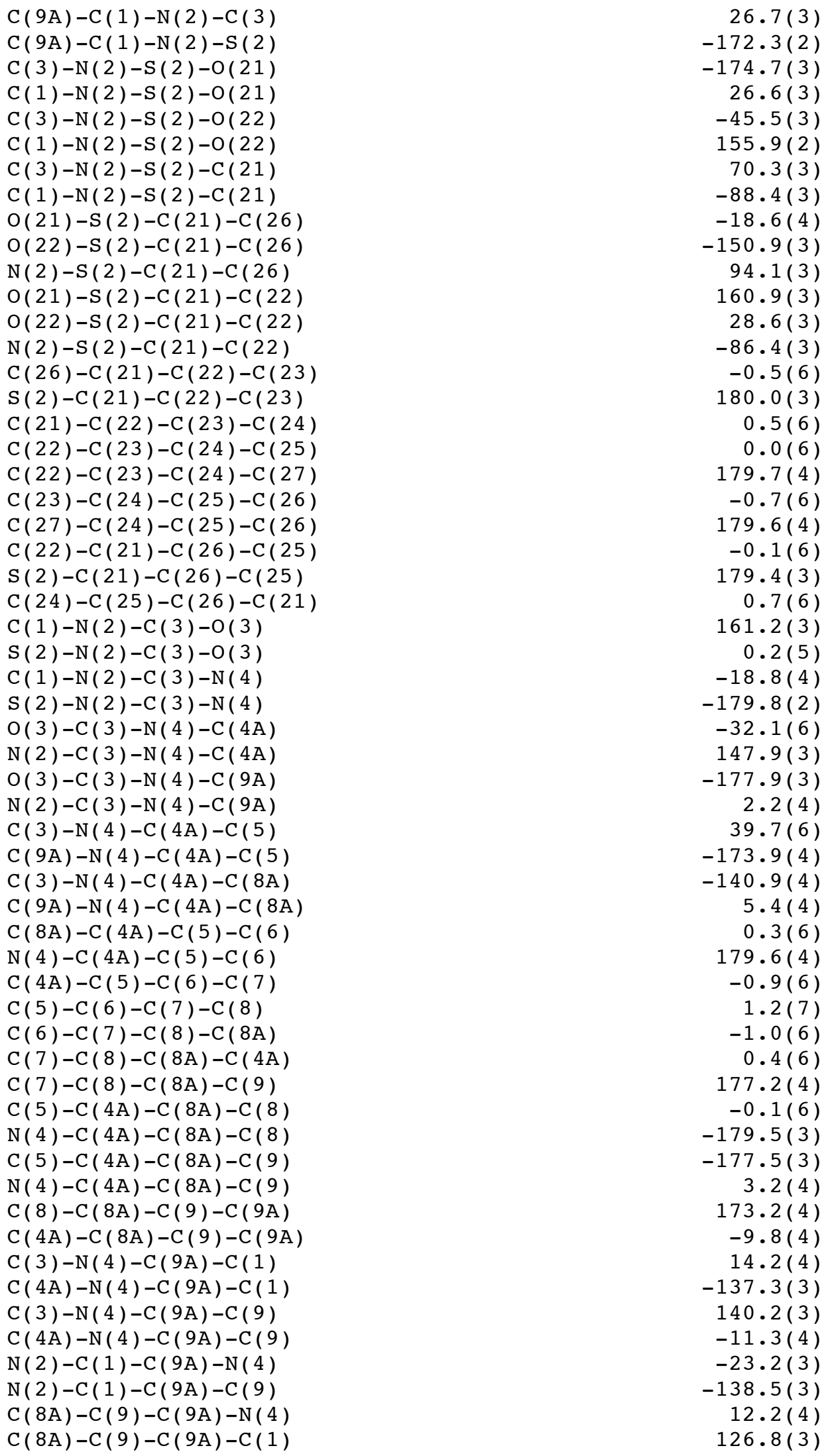


Table 7. Hydrogen bonds for $2 \mathrm{a}$ [A and deg.].

\begin{tabular}{lllll}
\hline D-H...A & d(D-H) & d(H...A & $d(D \ldots A)$ & $<(D H A)$ \\
C $(25)-\mathrm{H}(25) \ldots \mathrm{Cl}(1) \# 1$ & 0.95 & 2.82 & $3.679(7)$ & 150.5 \\
$\mathrm{C}(26)-\mathrm{H}(26) \ldots \mathrm{O}(22) \# 1$ & 0.95 & 2.56 & $3.369(5)$ & 143.5 \\
$\mathrm{C}(1)-\mathrm{H}(1 \mathrm{~B}) \ldots \mathrm{O}(21) \# 2$ & 0.99 & 2.64 & $3.233(4)$ & 118.8 \\
$\mathrm{C}(1)-\mathrm{H}(1 \mathrm{~B}) \ldots \mathrm{O}(22) \# 3$ & 0.99 & 2.60 & $3.237(4)$ & 122.3 \\
\end{tabular}

Symmetry transformations used to generate equivalent atoms:

$\# 1 \mathrm{x}+1, \mathrm{y}, \mathrm{z} \quad \# 2-\mathrm{x}+1,-\mathrm{y}+1,-\mathrm{z} \quad \# 3-\mathrm{x},-\mathrm{y}+1,-\mathrm{z}$ 


\section{Diamination of deuterated material 3:}

Trans-deuterated urea $\mathbf{3}$ was prepared using the selectively labelled allylic bromide which was obtained through hydrozirconation with the Schwartz reagent followed by subsequent quenching with $\mathrm{D}_{2} \mathrm{O}$ as described previously [D. Orain, J.-C. Guillemin, J. Org. Chem. 1999, 64, 3563]. Urea 3 was then elaborated via a sequence as described above for the unlabelled compound.<smiles>[2H]/C=C/CC(C)(C)CNC(=O)N[TeH2]</smiles>

${ }^{1} \mathrm{H} \mathrm{nmr}\left(300 \mathrm{MHz}, \mathrm{CDCl}_{3}\right): \square=0.82$ (s, 6H), 1.88 (dd, $\left.J=1.3,7.5 \mathrm{~Hz}, 2 \mathrm{H}\right), 2.42$ (s, 3H), 3.95 $(\mathrm{d}, J=6.0 \mathrm{~Hz}, 2 \mathrm{H}), 5.00(\mathrm{dt}, J=1.3,17.0 \mathrm{~Hz}, 1 \mathrm{H}), 5.75(\mathrm{dt}, J=7.5,17.0 \mathrm{~Hz}, 1 \mathrm{H}), 6.63(\mathrm{t}, J=$ $6.0 \mathrm{~Hz}, 1 \mathrm{H}), 7.29$ (d, $J=8.4 \mathrm{~Hz}, 2 \mathrm{H}), 7.78$ (d, $J=8.4 \mathrm{~Hz}, 2 \mathrm{H}), 9.27$ (br s, 1H).

${ }^{13} \mathrm{C} \mathrm{nmr}\left(75 \mathrm{MHz}, \mathrm{CDCl}_{3}\right): \square=21.54,24.67,34.58 .44 .06,49.67,117.50(\mathrm{t}, J=24.0 \mathrm{~Hz})$, $126.87,129.83,134.14,136.89,144.61,152.34$.

\section{Hexahydro-1-deutero-6,6-dimethyl-2-(p-tolylsulfonyl)pyrrolo-[1,2-e]imidazol-3-one}<smiles>[2H][13CH]1[C@H]2CC(C)(C)CN2C(=O)N1[13CH3]</smiles>

Synthesized according to procedure A.

${ }^{1} \mathrm{H} \mathrm{nmr}\left(300 \mathrm{MHz}, \mathrm{CDCl}_{3}\right): \square=0.96$ (s, 3H), 1.05 (s, 3H), 1.27 (dd, $J=12.4 \mathrm{~Hz}, J=9.2 \mathrm{~Hz}$, $1 \mathrm{H}), 1.78(\mathrm{dd}, J=12.4 \mathrm{~Hz}, J=6.1 \mathrm{~Hz}, 1 \mathrm{H}), 2.40(\mathrm{~s}, 3 \mathrm{H}), 3.76(\mathrm{~d}, J=11.3 \mathrm{~Hz}, 1 \mathrm{H}), 3.28(\mathrm{~d}, J$ $=11.3 \mathrm{~Hz}, 1 \mathrm{H}), 3.92(\mathrm{ddd}, J=5.9,8.6,8.8 \mathrm{~Hz}, 1 \mathrm{H}), 4.03(\mathrm{~d}, J=8.6 \mathrm{~Hz}, 1 \mathrm{H}), 7.30(\mathrm{~d}, J=8.4$ $\mathrm{Hz}, 2 \mathrm{H}), 7.89$ (d, $J=8.4 \mathrm{~Hz}, 2 \mathrm{H})$. 

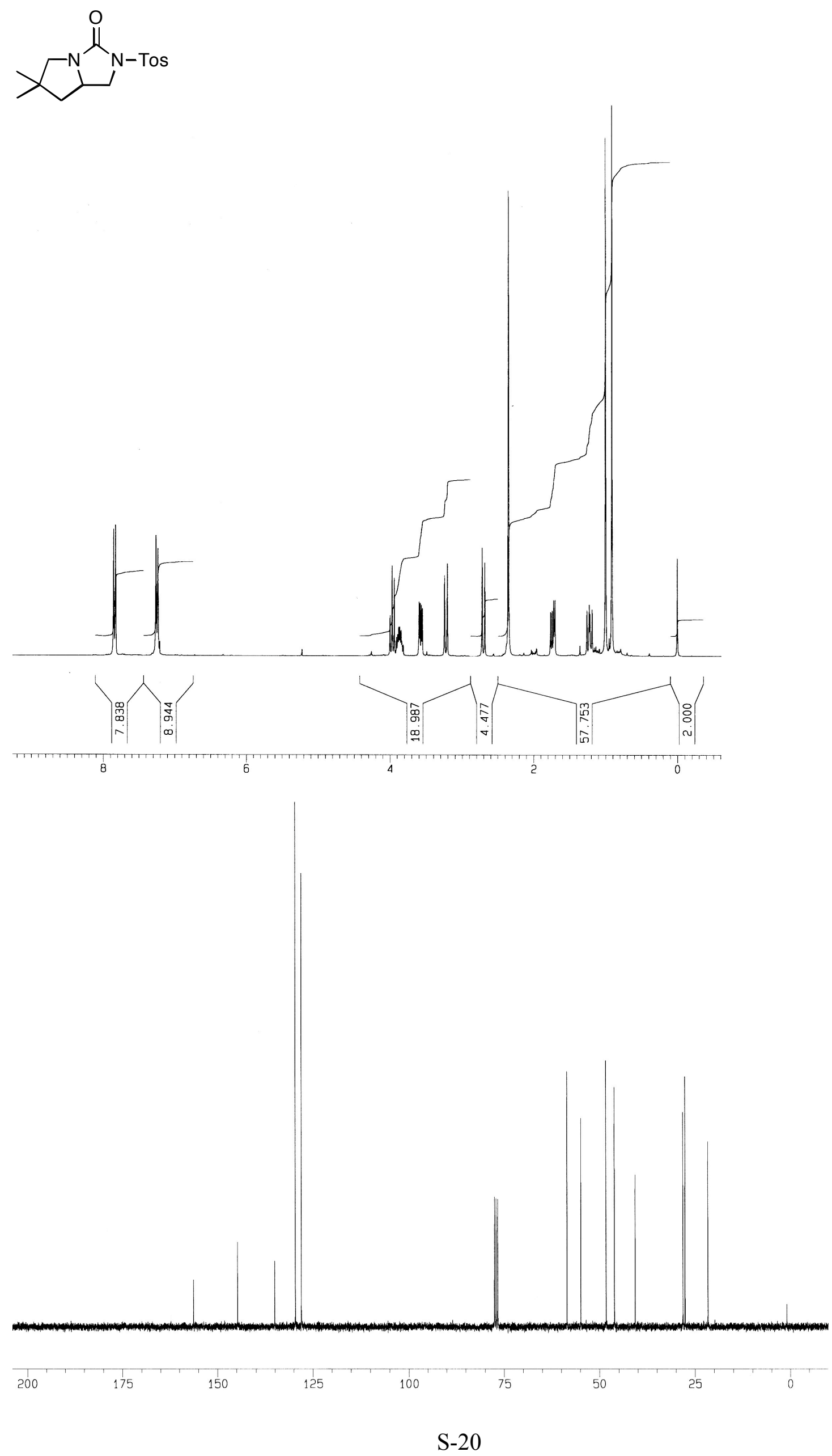
$\overbrace{}^{\mathrm{O}}$
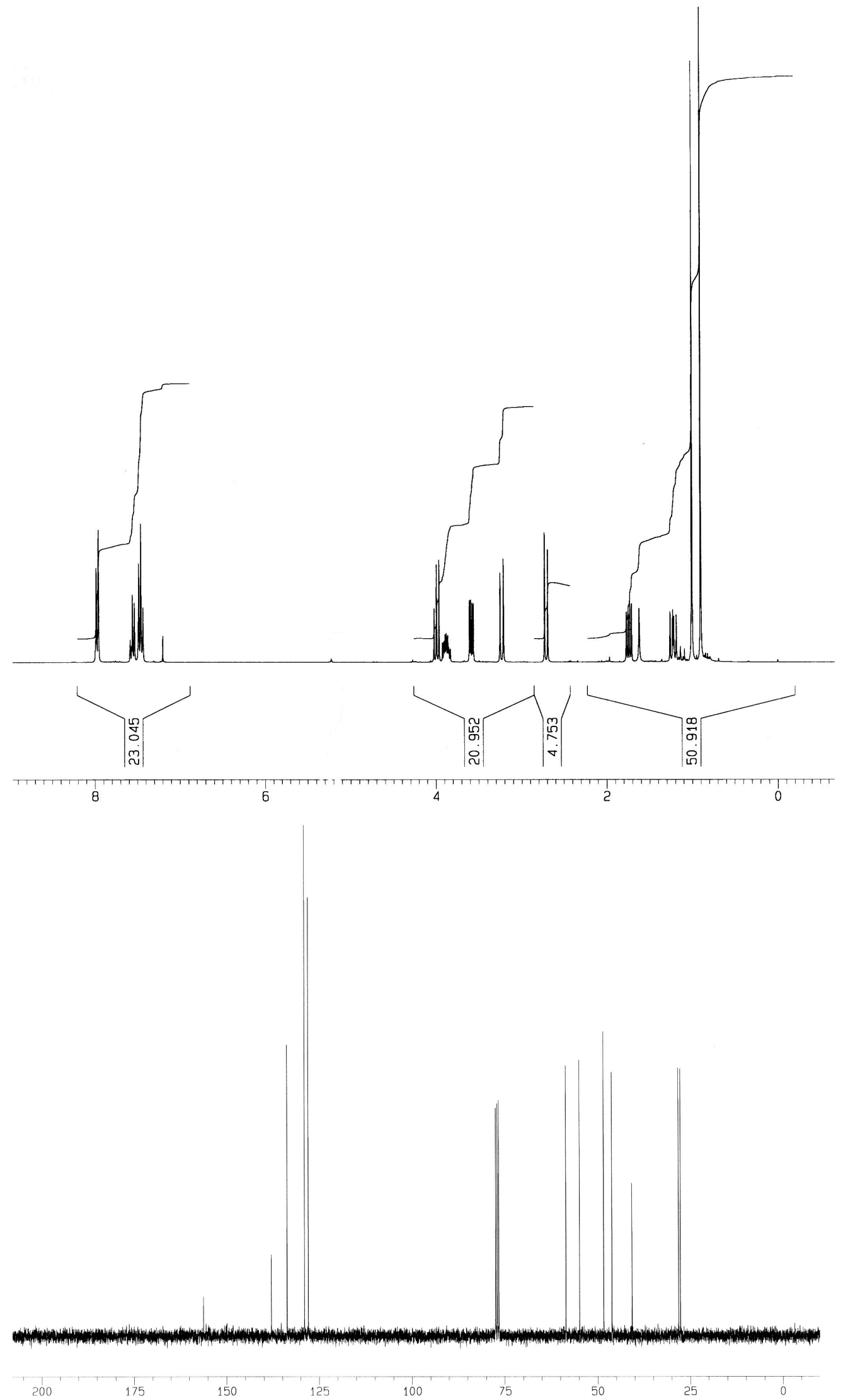

S-21 

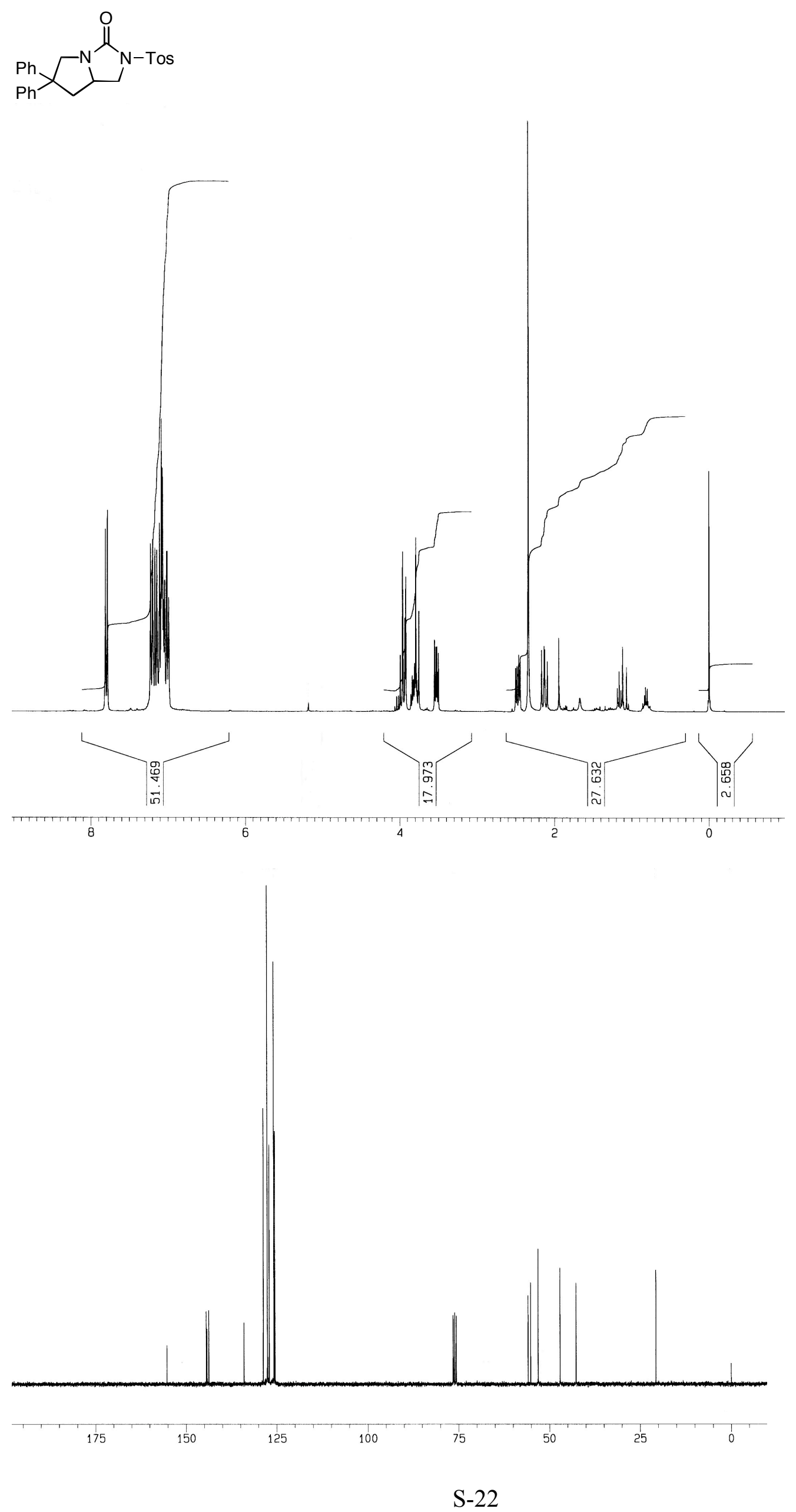

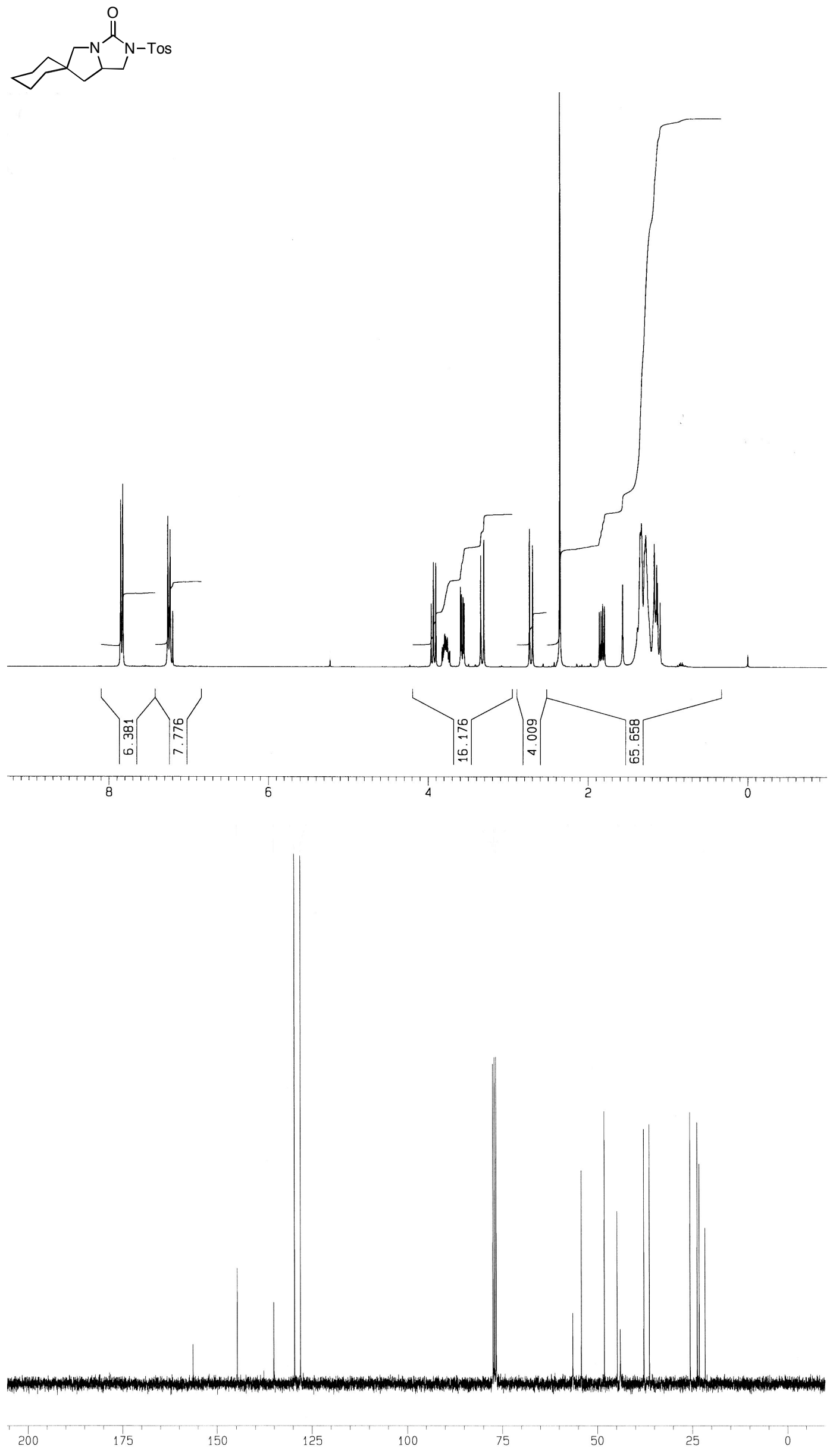

S-23 


$$
r^{\mathrm{O}-\mathrm{Tos}}
$$
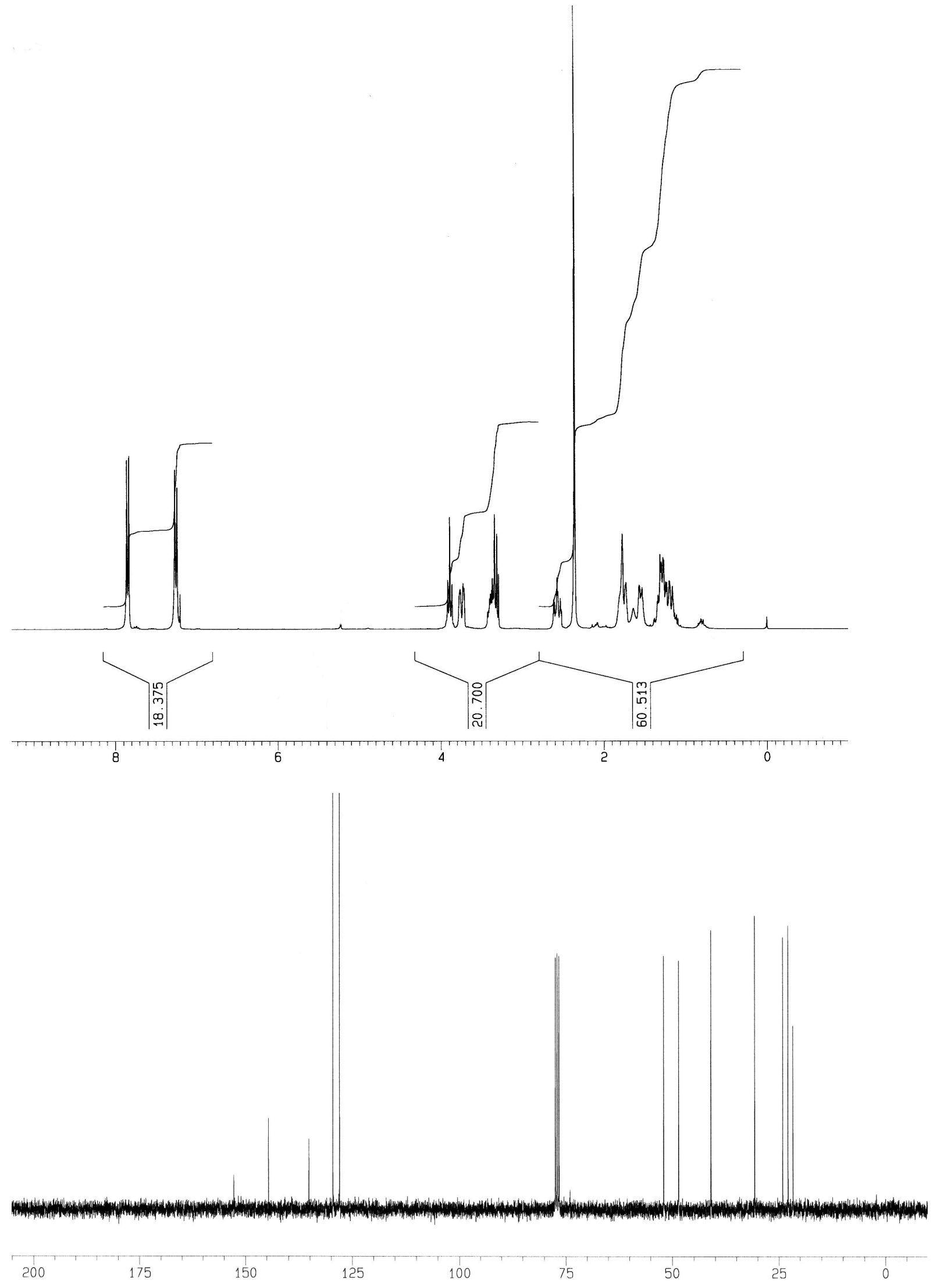

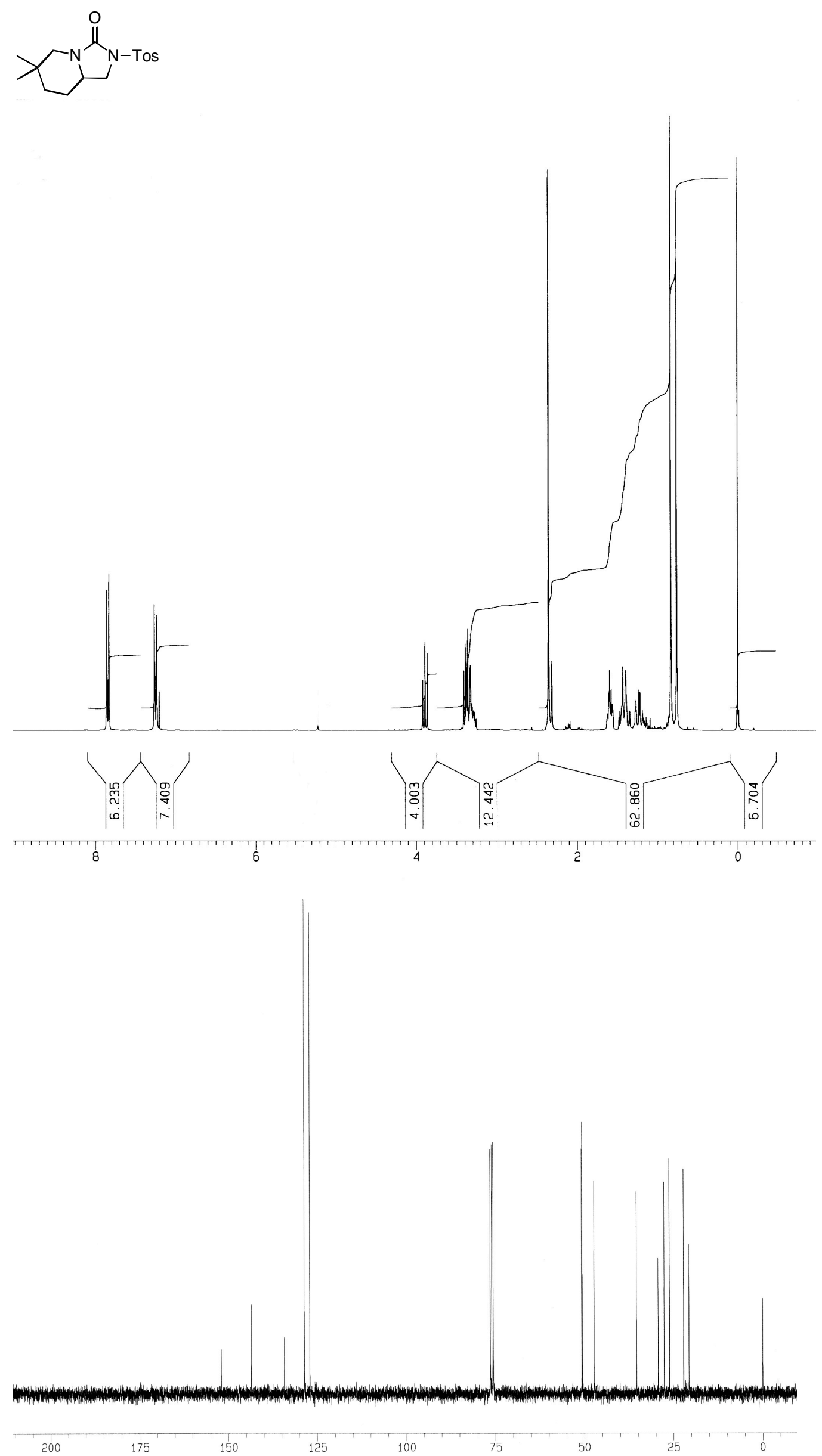

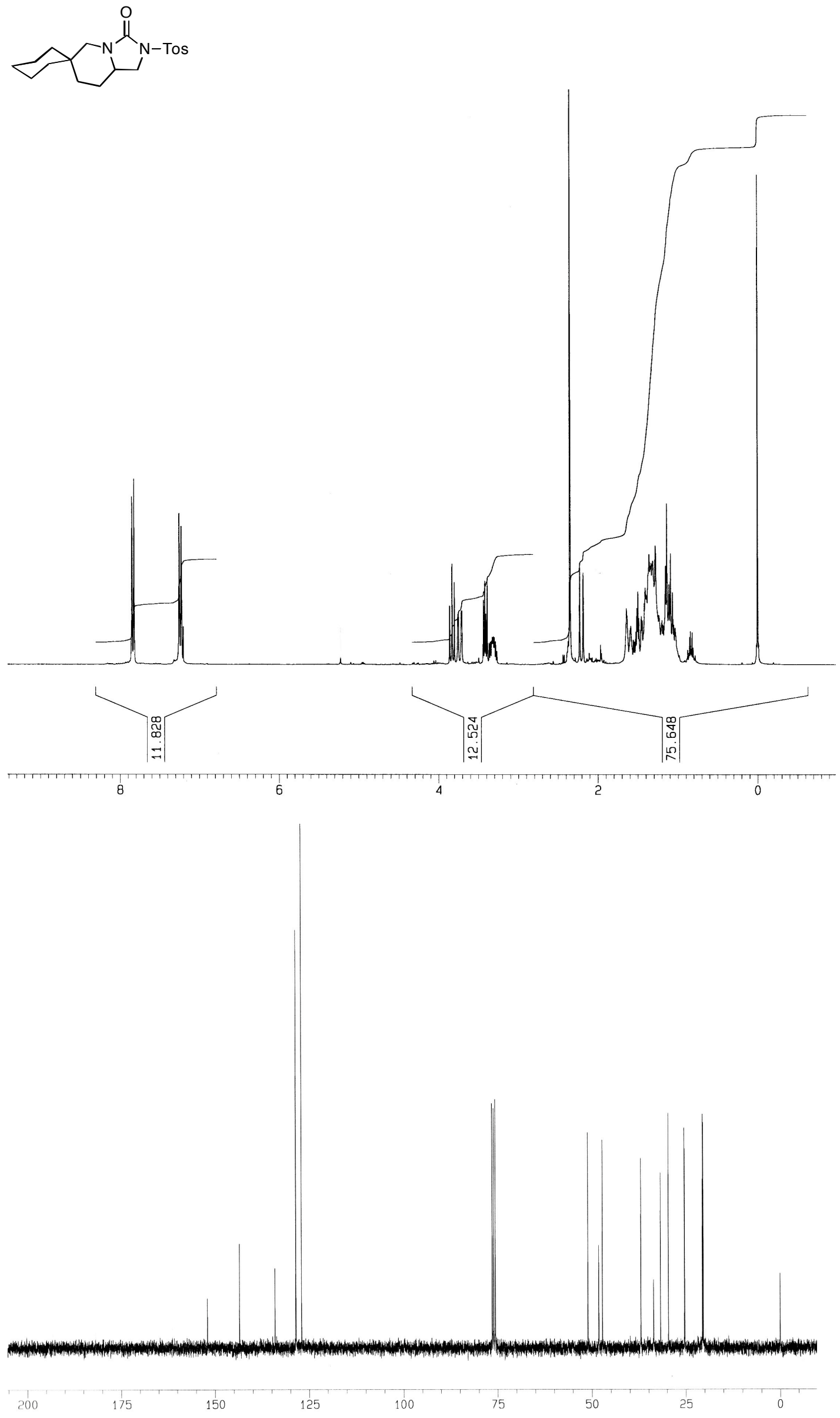

S-26 
<smiles>CC1(C)CN2C(=O)N([18O])CC2(C)C1</smiles>
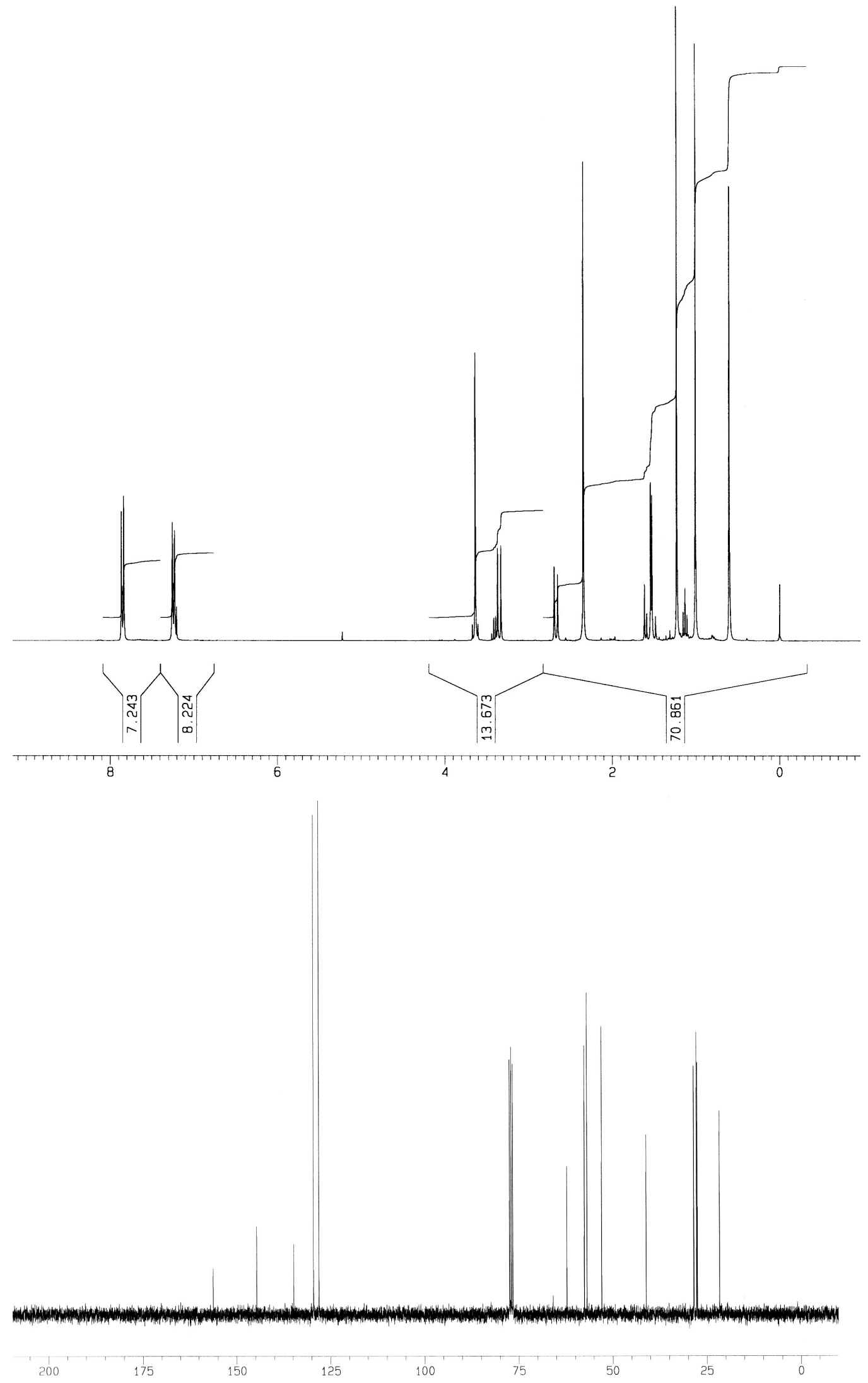
<smiles>O=C1N([18O])CC2Cc3ccccc3N12</smiles>
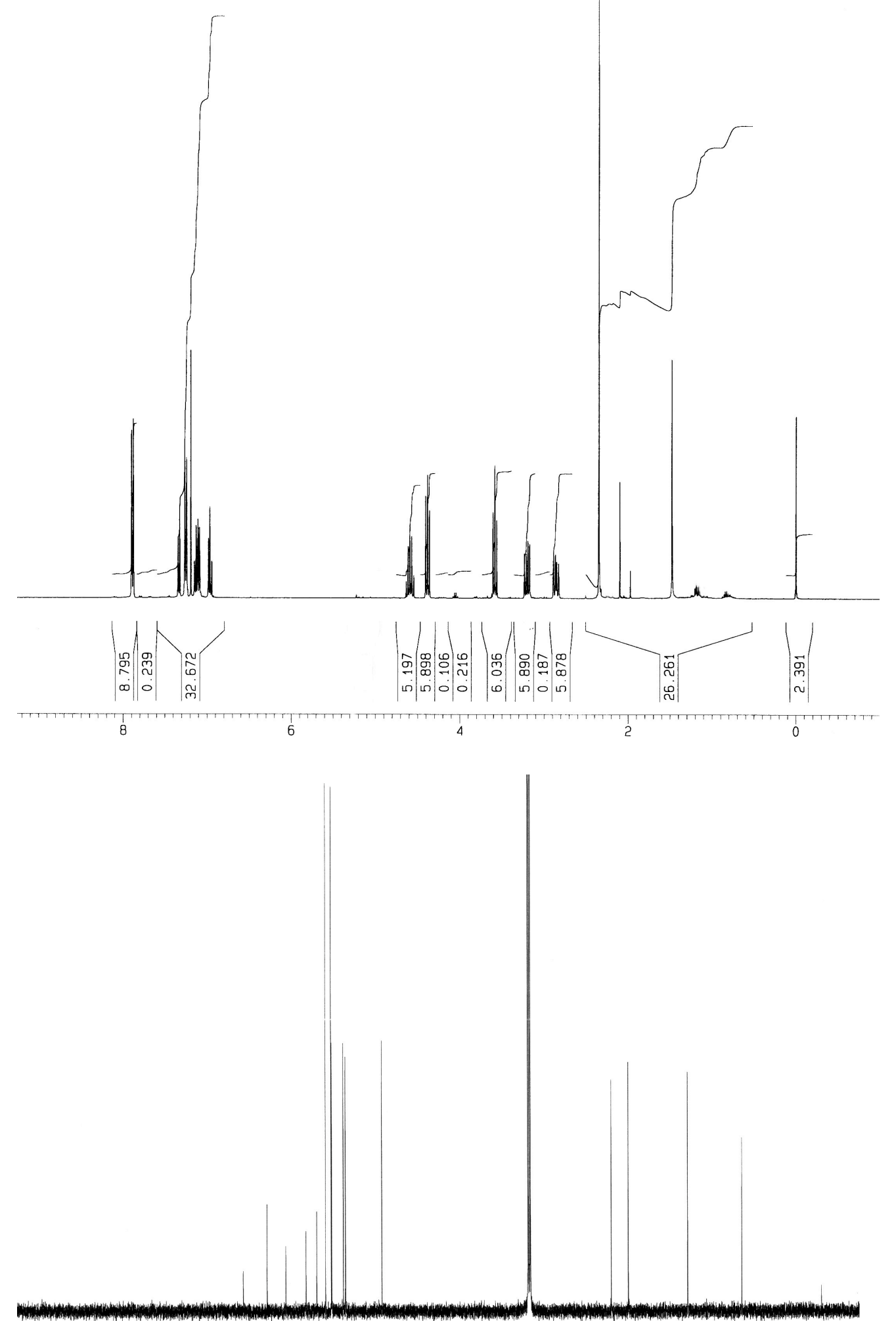

200 

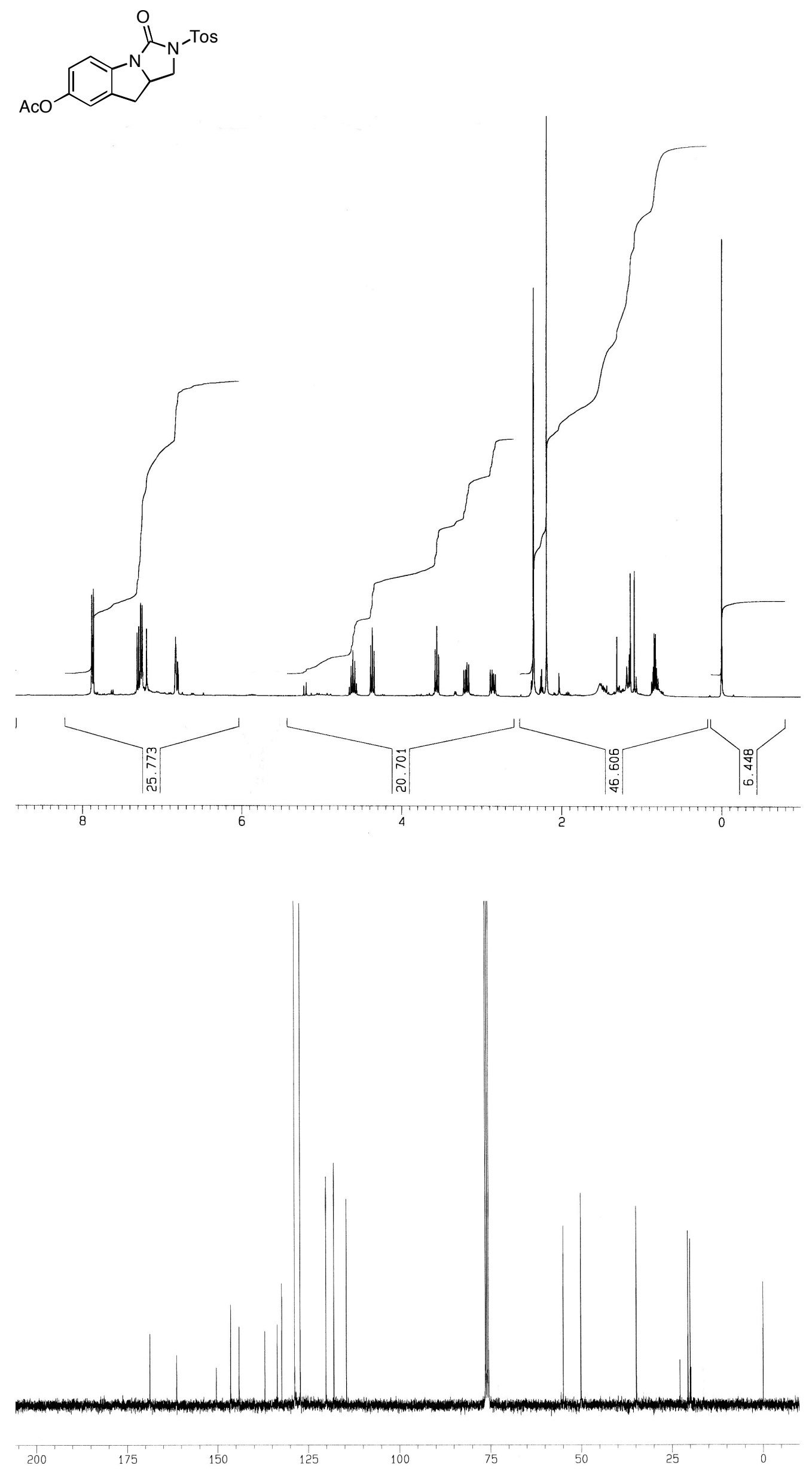